\title{
Blind Adaptive Chromatic Dispersion Compensation and Estimation for DSP-Based Coherent Optical Systems
}

\author{
Raffaele Corsini, Andrea Peracchi, Emma Matarazzo, Tommaso Foggi, Jeroen Nijhof, Gianluca Meloni,
} Luca Potì, Roberto Magri, and Ernesto Ciaramella, Senior Member, IEEE

\begin{abstract}
We propose an accurate and low-complexity blind adaptive algorithm for chromatic dispersion (CD) compensation and estimation in coherent optical systems. The method is based on a Frequency Domain Equalizer (FDE), a low complexity Time Domain Equalizer arranged in a butterfly structure (B-TDE) and an Optical Performance Monitoring (OPM) block in a loop configuration. The loop is such that, at each iteration, the CD value compensated by the B-TDE and estimated by the OPM is given to the FDE; according to this estimation, in the subsequent iteration, the FDE compensates also this quantity. The procedure is repeated until the majority of $C D$ is compensated by the FDE and a small residual quantity is compensated by a low complexity B-TDE with a small number of taps. The method is extended to long haul uncompensated links exploiting the information on the mean square error (MSE) provided by the B-TDE. The proposed algorithm is then experimentally validated for a polarization multiplexed quadrature phase shift keying (PM-QPSK) signal at $112 \mathrm{Gbit} / \mathrm{s}$ propagating along $1000 \mathrm{~km}$ of uncompensated $Z$ PLUS ${ }^{\circledR}$ optical fiber. A statistical analysis of the performance of the proposed solution, in terms of mean value and standard deviation of the $C D$ estimation error, is carried out, running a set of simulations including different impairments, such as noise, polarization dependent loss, polarization mode dispersion and self-phase modulation in a line of $1000 \mathrm{~km}$ of uncompensated G.652 optical fiber. Our method could be used to compensate and estimate any $C D$ quantity without increasing the number of taps in the B-TDE and exploiting devices already included in the system (TDE, FDE and OPM) arranged in a loop.
\end{abstract}

Index Terms-Chromatic dispersion compensation, frequency domain equalizer, optical performance monitoring, polarization multiplexing, QPSK, time domain equalizer.

\section{INTRODUCTION}

$\mathbf{O}$ PTICAL transmission systems based on coherent detection and digital signal processing (DSP) of polarization division multiplexing (PDM) multilevel modulation formats are

Manuscript received June 15, 2012; revised January 18, 2013; accepted May 01, 2013. Date of publication May 07, 2013; date of current version June 05, 2013. This work was supported in part by the Regione Toscana through the ARNO T3 project, POR CReO FESR 2007-2013, PAR FAS 2007-2013.

R. Corsini, A. Peracchi, G. Meloni, and E. Ciaramella are with Scuola Superiore Sant'Anna, 56124 Pisa, Italy. They are also with the Italian National Consortium for Telecommunications (CNIT), 56124 Pisa, Italy (e-mail: r.corsini@ sssup.it; a.peracchi@sssup.it; g.meloni@sssup.it; e.ciaramella@sssup.it).

E. Matarazzo was with Scuola Superiore Sant'Anna, Pisa 56124, Italy. She is now with Space Engineering S.p.A. 91, Rome 00155, Italy (e-mail: emma. matarazzo@space.it).

T. Foggi and L. Potì are with CNIT, I-43100 Parma, Italy (e-mail: Tommaso. foggi@cnit.it; luca.poti@enit.it).

J. Nijhof and R. Magri are with Ericsson Telecomunicazioni, 16152 Genova, Italy (e-mail: jeroen.nijhof@ericsson.com; roberto.magri@ericsson.com).

Digital Object Identifier 10.1109/JLT.2013.2262211 attracting strong attention and are considered for nextgeneration optical networks [1]. The main advantage of coherent receivers is to provide both amplitude and phase information of the received signal. These allow the DSP equalizer to compensate for the channel linear transfer function in order to recover the transmitted signal. Hence, the digital equalizer structure compensates for all linear channel impairments, namely uncompensated chromatic dispersion (CD) and polarization mode dispersion (PMD) [2], [3]. This is particularly true in the weakly non-linear regime of typical optical systems.

In a first approach, both CD and PMD are compensated by a time-domain equalizer (TDE) [4], implemented by means of four finite impulse response (FIR) filters arranged in a butterfly configuration (B-TDE). In case of uncompensated links, the intersymbol interference (ISI) is mainly due to the accumulated $\mathrm{CD}$ and this value determines the required number of taps in the B-TDE [5], [6]. In high speed systems (100 Gb/s and beyond), even in the case of dispersion managed links, the ISI induced by the residual $\mathrm{CD}$ is relevant since the corresponding channel memory is a quadratic function of the baud rate [7].

Another approach assumes that the equalizer is made of two stages: a non-adaptive finite impulse response filter (FIR) of proper length dedicated to perform a coarse compensation of the static accumulated $\mathrm{CD}$ and a short adaptive B-TDE dedicated to PDM signals de-multiplexing and PMD compensation [8]. This allows a reduced complexity B-TDE (low number of taps), with fast update for tracking time-varying effects like PMD and rotation of the state of polarization (SOP). However for a large amount of uncompensated $\mathrm{CD}$, it is convenient to replace the FIR with a frequency-domain equalizer (FDE) [9]-[11]. In the frequency domain the FDE multiplies the signal by the inverse of the CD transfer function, which is an all-pass filter with a quadratic phase. The advantage of the FDE with respect to the FIR is that the computational complexity of a FDE is lower even if compared to a FIR with a small number of taps [12]. However both these solutions require the knowledge of the residual $\mathrm{CD}$ value of the link. In many cases, the $\mathrm{CD}$ is assumed as a known quantity and preset in the FDE [2], [8]. Particularly, in links without dispersion compensation, accurate $\mathrm{CD}$ knowledge is critical, since it allows relaxing the condition for the subsequent equalization performed by the B-TDE. In this case, an appropriate compensation at the FDE level is needed otherwise the digital coherent receiver can fail completely [13]. A blind approach without large overhead is preferred in order to prevent any loss of capacity. Moreover, even if the CD parameter does 
not change over time and estimation is only required for initialization of the equalizer, this procedure must be iterated each time the lightpaths are established in a dynamic transparent optical network

Recently, the problem of the adaptive CD compensation in dynamically switched lightpaths was addressed using delay tap sampling technique [14], minimum mean-square error (MMSE) equalizer assisted by channel estimation from a training sequence [15], overlap frequency domain equalizer (OFDE) with a blind estimation algorithm operating in time or frequency domain [10], [16], [17], or using the time domain least mean square (LMS) adaptive filters [18]. The main characteristics of the solutions presented in literature are: the need of a look-up table [14], [16], [17], the need of a training sequence [15] and a block for only $\mathrm{CD}$ estimation [10]. Moreover $\mathrm{Xu}$ et al. [18] analyzed three different solutions: TDE LMS filter, FIR filter and blind look up filter. The first two solutions have the characteristics of high number of taps needed for an effective CD compensation, whilst the last needs a look-up table.

Recently, an Optical Performance Monitoring (OPM) algorithm for coherent optical receivers based on the elaboration of the B-TDE taps coefficients has been introduced [19], [20] in order to provide fiber linear parameters information in a simple, cost- and power-effective way. However, this technique has an intrinsic limitation, i.e., when the value of the uncompensated $\mathrm{CD}$ induces an ISI length that could not be compensated by a B-TDE with an acceptable complexity (i.e., a number of taps not exceeding the number of 21), the estimation of the CD is not reliable [19].

In this work we propose a blind and adaptive CD compensation and estimation technique that exploits the best characteristics of both B-TDE and FDE together with an auxiliary OPM block. The proposed method aims at exploiting elements already present in the system (TDE, FDE and OPM), and can be applied to networks where the propagation distance can switch dynamically [13]. The required number of B-TDE taps for both CD compensation and estimation does not depend on the accumulated CD $D$ during propagation, thanks to the iterative loop algorithm. Hence, our technique is robust to any amount of accumulated $\mathrm{CD}$, without the need of additional resources (expressed in terms of B-TDE taps number) just increasing the number of iterations in the loop algorithm.

We tested the performance of our solution in terms of mean value and standard deviation of the $\mathrm{CD}$ estimation error running a set of simulations where different types of impairments such as optical noise, polarization dependent loss (PDL), PMD and self-phase modulation were added to a line of $1000 \mathrm{~km}$ of uncompensated G.652 fiber.

This paper is structured as follows. Section II provides a description of the OPM block, shows the potentiality and the related problems; Section III presents the operation principle of the proposed solution and Section IV reports its experimental validation. In Section $\mathrm{V}$ a statistical analysis of the proposed method is illustrated reporting the mean value and the standard deviation of the CD estimation error in presence of different fiber impairments. In Section VI we analyzed the computational complexity of the method and in Section VII we draw the conclusions.

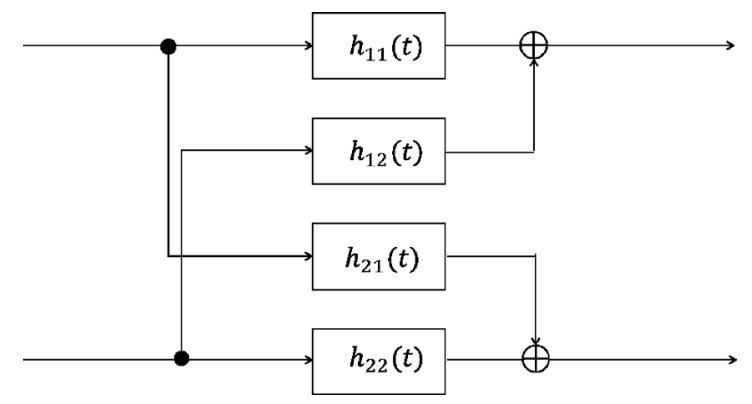

Fig. 1. TDE structure in a FIR butterfly configuration (B-TDE).

\section{OPM ALGORITHM}

\section{A. Theoretical Description}

A Single Mode Fiber (SMF) can be represented as a twoinput/two-output channel, whose frequency response $\mathbf{H}_{c h}(f)$ is represented by a $2 \times 2$ Jones matrix, accounting for both $C D$ and PMD [5]. A two-dimensional (2-D) matched filter can thus achieve perfect compensation of the corresponding phase distortions (see [21] and references therein). An adaptive time domain 2-D fractionally-spaced feed-forward equalizer (FFE), in a B-TDE structure, implements the 2-D matched filter if the MMSE criterion [22] for the adaptation of its coefficients is adopted [21]. Hence, the core of the DSP in polarization-multiplexed coherent optical systems is constituted by the B-TDE implemented in a butterfly structure of adaptive complex-valued FIR filters with impulse response equal to $h_{i j}(t)$ (Fig. 1). This scheme also allows to demultiplex two PDM signals. The required number of taps of the B-TDE is governed mainly by the maximum residual uncompensated $\mathrm{CD}$ and marginally by the mean differential group delay (DGD) [5], [7].

The taps of the B-TDE filter used in this work were calculated using the method described in [21] and references therein, based on an asynchronous detection strategy and asynchronous filter adjustment algorithm, derived from wireless communications. This technique was demonstrated to be effective to compensate also for both the transmitter and receiver lasers phase noise [21]. The taps were adaptively updated accordingly to the constant modulus algorithm (CMA) for initial convergence and then using the decision-directed least mean square (DD-LMS) algorithm for tracking the time varying polarization distortions. Both algorithms typically converge to the MMSE solution.

If the number of taps is sufficiently large (greater or equal to the channel memory), the B-TDE perfectly compensates for $\mathrm{CD}$ and PMD and its frequency response $\mathbf{H}_{B-T D E}(f)$ is a good approximation of the inverse of the channel frequency response [21]. The perfect channel inversion cannot be performed due to the presence of a noise factor [21]. The two-dimensional equalizer coefficients are represented by a $2 \times 2$ complex matrix, ranging over the equalizer length [21]:

$$
\mathbf{H}_{B-T D E}(f)=\left(\begin{array}{ll}
H_{11}(f) & H_{12}(f) \\
H_{21}(f) & H_{22}(f)
\end{array}\right) \approx \mathbf{H}_{c h}^{-1}(f),
$$

where $H_{i j}(f)$ are the Fourier transform of $h_{i j}(t)$ and thus represent the frequency response of each FIR filter composing the adaptive 2-D fractionally-spaced FFE (Fig. 1), obtained by the 
Fourier transform of the B-TDE taps. For simplicity, we use a frequency-continuous notation in the following, but in the practice both the filters and their spectra are given by their discrete samples. In the linear regime, both $\mathrm{CD}$ and PMD contribute to the total channel impulse response; using the unitary properties of the fiber transfer function [23], they can be separated by proper calculations [20], [24]:

$$
\mathbf{H}_{c h}(f) \approx A(f) \cdot \mathbf{U}(f),
$$

where $A(f)$ is a polarization independent linear function with quadratic phase and is related to the CD. It includes any band-pass optical filtering and any low-pass electrical filtering; $\mathbf{U}(f)$ is a frequency dependent unitary matrix and represents all the polarization effects like PMD and polarization dependent loss (PDL). The B-TDE compensates also for other channel impairments; if we consider only the all-pass transfer function responsible for residual $\mathrm{CD}$ thus avoiding filtering effects, the estimated $A(f)$ is related to $\mathbf{H}_{B-T D E}(f)$ by the following expression:

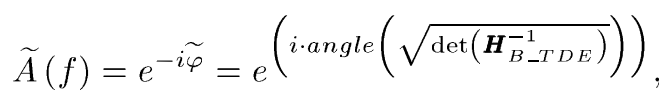

Where $2 \tilde{\varphi}=\beta_{2} L \omega^{2}, L$ is the transmission distance, $\omega$ is the angular frequency and $\beta_{2}$ is the dispersion parameter. So we can then estimate the channel $\mathrm{CD}(D)$ by the following equation:

$$
\widetilde{D}=\left\langle-\frac{2 \pi c}{\lambda^{2}} \frac{\partial^{2} \widetilde{\varphi}}{\partial \omega^{2}}\right\rangle\left[\frac{\mathrm{ps}}{\mathrm{nm}}\right],
$$

where $\langle\cdot\rangle$ indicates averaging over the signal bandwidth.

\section{B. Numerical Validation}

First we tested the OPM by numerical simulations, setting different values of $D$ without FDE dispersion compensation. The considered signal was $112 \mathrm{Gbit} / \mathrm{s}$ polarization multiplexed quadrature phase shift keying with differential encoding (PM-DQPSK). A non-return-to-zero (NRZ) pulse filtered through an electrical baseband fourth-order Gaussian filter with $-3 \mathrm{~dB}$ bandwidth equal to $0.8 / T$ ( $T$ is the symbol interval) was adopted at the transmitter. Two fourth-order Gaussian optical filters with $3-\mathrm{dB}$ bandwidth equal to $1.8 / T$ and $1.6 / T$ were present at transmitter and receiver end, respectively. The post-detection electrical filter was a fifth-order Bessel with 3 -dB bandwidth equal to $0.8 / T$. The transmitted signal also experienced optical amplification before the receiver. The low-pass equivalent of the amplified spontaneous emission (ASE) noise can be modeled as two independent complex noise components, each with two-sided power spectral density (PSD) equal to $N_{0}$, taking into account the noise components on two orthogonal states of polarization. The optical signal-to-noise ratio (OSNR) was set to $17.5 \mathrm{~dB}$, which corresponds to around $1 \cdot 10^{-4}$ bit error rate. The receiver exploited the homodyne coherent detection scheme, without local oscillator frequency offset and phase noise in order to include only the effect of CD on the B-TDE taps.

Given the above channel and receiver configurations, we tested the convergence time $\left(T_{c}\right)$ of the B-TDE, measured in symbol periods $T=1 / R_{b}$, where $R_{b}$ is the symbol rate.
TABLE I

Channel Memory $\left(N_{t o t}\right)$ And ConVergence Time $\left(T_{c}\right)$ Measured IN SYMBOL INTERVAL FOR DIFFERENT VALUES OF UNCOMPENSATED $D$.

\begin{tabular}{|c|c|c|c|c|}
\hline $\begin{array}{c}D \\
\mathrm{ps} / \mathrm{nm})\end{array}$ & $N_{\text {tot }}$ & $T_{c}(5$ taps $)$ & $T_{c}(11$ taps $)$ & $T_{c}(23$ taps $)$ \\
\hline 0 & 1 & 500 & 1400 & 1900 \\
\hline 188 & 3 & 700 & 1600 & 2100 \\
\hline 533 & 5 & 6500 & 1800 & 2300 \\
\hline 690 & 6 & 8400 & 2400 & 2600 \\
\hline 1130 & 9 & -- & 6500 & 3100 \\
\hline 1380 & 11 & -- & 9400 & 3400 \\
\hline
\end{tabular}

We used the DD-LMS algorithm to implement the MMSE criterion for the adaptation of the B-TDE coefficients. In order to estimate the convergence time, we decided that, according to the usual choice, the convergence had been reached when the MSE value had fallen below the $120 \%$ of its final steady-state value. At initial condition, all the equalizer coefficients were set to zero but the central tap, set to 1 . The MSE was computed averaging over a sliding window of $n=100$ symbols.

A key parameter in our case is the channel memory of the link. As known, a channel having uncompensated dispersion $\beta_{2} L$ ( $L$ is the link length) has a channel memory of $N_{c d}$ symbols due to CD approximately given by [6]:

$$
N_{C D} \approx 2 \pi\left|\left(\beta_{2} L\right)\right| R_{b}^{2}
$$

If the PMD is not included, the total channel memory is given by $N_{t o t}=N_{C D}+N_{f}$, where $N_{f}$ is the memory contribution due to optical and electrical filtering. In our case $N_{f} \approx 1$. The required number of taps in the B-TDE is $N>\eta N_{t o t}$ where $\eta$ is the oversampling factor (in our case $\eta=2$ ).

The results are summarized in Table I where the channel memory $N_{t o t}$ in terms of symbol periods is reported together with the convergence time $T_{c}$ is given in terms of number of symbol periods; for each value of uncompensated $\mathrm{CD}$, this $T_{c}$ parameter is reported for three different values of the number of taps in the $\operatorname{TDE}(5,11,23)$. We chose these last three values because they represent a reasonable case (11) and two limit conditions ( 5 and 23). Indeed a fractionally-spaced B-TDE with 5 taps is able to compensate a channel with $N_{t o t}=2$, which can be realistically induced simply by limited dispersion and common filtering, whilst 23 fractionally-spaced taps represent a too high number in terms of B-TDE implementation complexity [12]. In [25], it was observed that 9 taps are required for a DGD tolerance of $100 \mathrm{ps}$ at $112 \mathrm{~Gb} / \mathrm{s}$, and 5 taps are required for a DGD tolerance of $60 \mathrm{ps}$, thus confirming that 11 taps represent a good trade-off between complexity and DGD robustness, also maintaining a margin against residual ISI (e.g., due to filtering effects and residual uncompensated $C D$ ). When the equalizer cannot converge, we do not report any value of $T_{c}$ in Table I.

As expected, the B-TDE can converge even if $N_{t o t}$ is slightly greater than the equalizer length, but the convergence time increases drastically. In general if the equalizer length is around $2 \cdot N_{\text {tot }}$ or higher, a shorter equalizer converges with a smaller number of symbol intervals. In all cases, the step size for the equalizer taps was set to $1 \cdot 10^{-2}$; the equalizer steady-state performance and its convergence speed can be improved by optimizing the step-size during the taps convergence and adaptation process. 


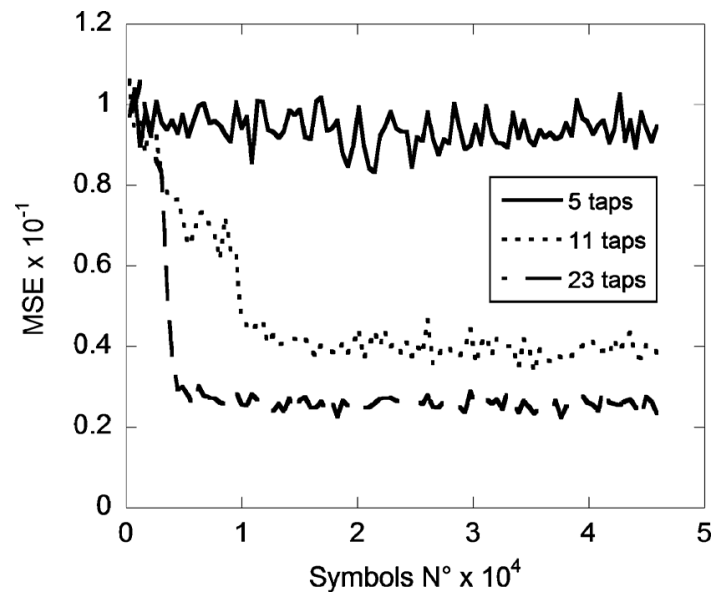

Fig. 2. MSE of the B-TDE with 5,11 and 23 taps $(D=1380 \mathrm{ps} / \mathrm{nm}$, OSNR $=17.5 \mathrm{~dB})$.

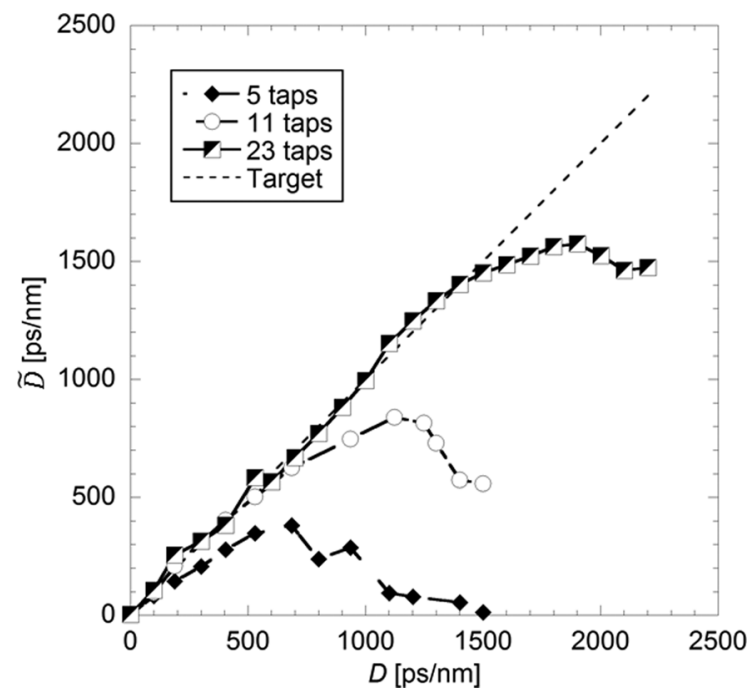

Fig. 3. OPM estimated CD ( $\widetilde{\boldsymbol{D}})$ vs. $D$ value. 5 taps (diamonds), 11 taps (circles) and 23 taps (black/white squares).

In Fig. 2 we report the MSE convergence curves as a function of the number of symbols at OSNR $=17.5 \mathrm{~dB}$, and $D=$ $1380 \mathrm{ps} / \mathrm{nm}$ for three different B-TDE lengths $(5,11$ and 23 taps).

From Fig. 2 we see that the MSE of the 5-taps B-TDE does not reach a steady-state value if the uncompensated $D$ is equal to $1380 \mathrm{ps} / \mathrm{m}$. The B-TDE with 23 taps reaches the convergence within only 3400 symbols whilst the case with 11 taps needs 9400 symbols to converge. Given that the B-TDE is fractionally spaced, we need a number of taps greater or equal to twice the channel memory; in the reported case, 11 taps are far below the channel memory (see Table I) and the steady-state MSE is not the minimum achievable. Once the number of taps is enough to compensate all channel ISI, the steady-state MSE reaches its minimum mean value.

After reaching the convergence the B-TDE taps configuration was passed to the OPM algorithm which exploits (4). In Fig. 3 we report estimated CD values $(\widetilde{D})$ vs. the uncompensated $D$ using B-TDE's having different number of taps. The B-TDE with a high number of taps (23) provides a good CD

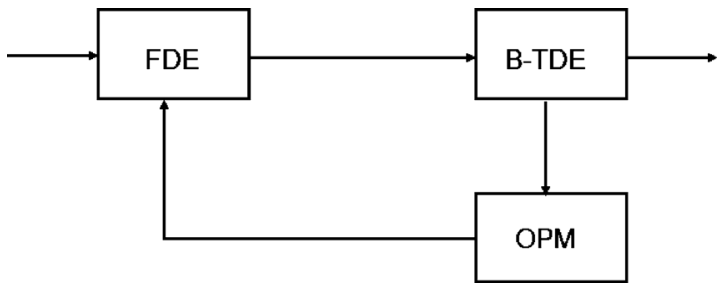

Fig. 4. Proposed loop solution for adaptive CD compensation, based on a FDE, a low complexity B-TDE and an OPM.

estimation up to a value of $1500 \mathrm{ps} / \mathrm{nm}$ with an error below 50 $\mathrm{ps} / \mathrm{nm}(3.3 \%)$. Increasing $D$ up to $2000 \mathrm{ps} / \mathrm{nm}$ the error reaches the value of $476 \mathrm{ps} / \mathrm{nm}(24 \%)$. Decreasing the number of taps to 11 , if $D=680$ we obtained an error of $60 \mathrm{ps} / \mathrm{nm}(8.8 \%)$ and an error of $190 \mathrm{ps} / \mathrm{nm}$ for $D=936 \mathrm{ps} / \mathrm{nm}(20 \%)$. We also tested the OPM performance with a B-TDE with a minimum number of taps (5). In this case we obtained an estimation error of 180 $\mathrm{ps} / \mathrm{nm}$ for $D=530 \mathrm{ps} / \mathrm{nm}(34 \%)$. The error increases up to $650 \mathrm{ps} / \mathrm{nm}$ for $D=936 \mathrm{ps} / \mathrm{nm}(70 \%)$. For higher values of uncompensated $D$ the B-TDE does not converge and the $\widetilde{D}$ value estimated is unreliable.

\section{LOOP OPERATION PRINCIPLE}

We have seen that the filter-coefficient based estimation (OPM) is a good technique to estimate the uncompensated CD, but the required number of taps for a good estimation increases with the accumulated CD.

For this reason we propose a solution in order to jointly estimate and compensate CD, still exploiting the OPM even in case of long-haul link with unknown and uncompensated CD. The proposed solution exploits a FDE, a low complexity B-TDE with a limited number of taps and an OPM. It allows to obtain at the same time a precise setting of the FDE together with a good $\mathrm{CD}$ estimation with a blind approach. The concept is to insert the FDE-based CD compensation block, a low complexity B-TDE and the OPM in a loop configuration as reported in Fig. 4. In this loop, the CD value estimated by the OPM provides a feedback to the FDE. In Fig. 5 the block diagram of the process flow of the proposed solution is presented.

It is composed of two loops. Loop 1 is used to coarsely set the FDE thus allowing the B-TDE and OPM to provide a reliable value of $\widetilde{D}$. Loop 2 performs a fine tuning of the FDE exploiting the $\widetilde{D}$ value estimated by the OPM.

At each iteration of Loop 1 the MSE value is compared to a threshold $M_{1}$ in order to decide if the convergence is close so that Loop 2 could be start. In Loop 2 the OPM estimates the dispersion $\widetilde{D}$ that is left uncompensated by the FDE; this value is then passed to the FDE whose dispersion is accordingly changed. The MSE value is also checked to establish when the iterative estimation and compensation process can be stopped. This procedure allows to maximize the amount of CD compensated by the FDE and minimize the amount of CD compensated by the B-TDE.

In the following we report a detailed description of the process flow showed in Fig. 5. The FDE is initialized to $D_{0}=0 \mathrm{ps} / \mathrm{nm}$ for a blind approach. If the CD accumulated by the signal during propagation in the network can range between 


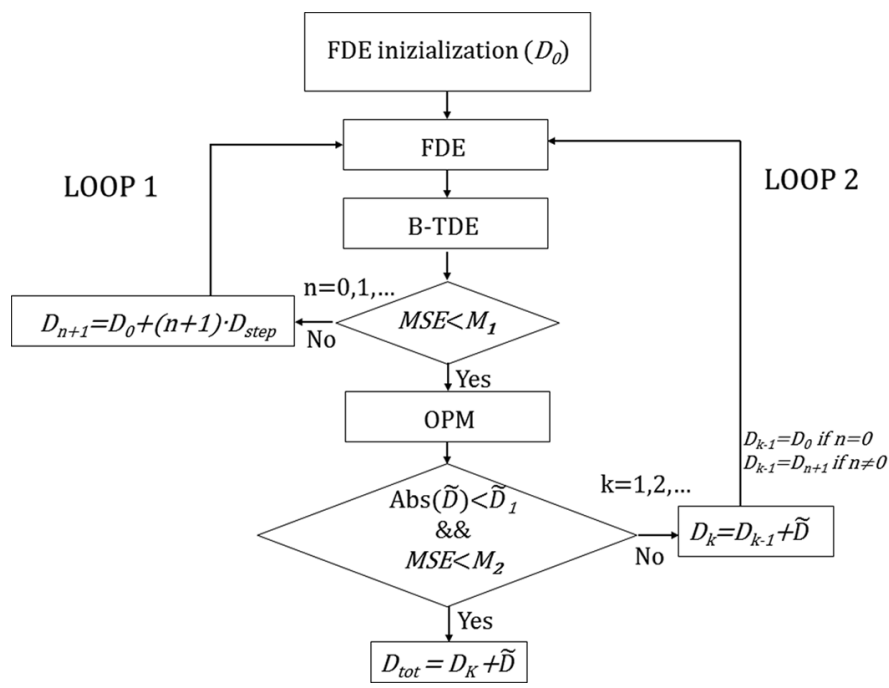

Fig. 5. Block diagram of the process flow for the proposed solution $(\mathrm{k}=\mathrm{K}$ in the last iteration of Loop2).

a known minimum $\left(D_{\min }\right)$ and maximum value, the FDE can be initialized to $D_{\min }$. After this initialization, FDE and B-TDE are applied (Loop 1 in Fig. 5); then the MSE value of the B-TDE is compared to a threshold $\left(M_{1}\right)$. If the MSE is higher than $M_{1}$, the estimated $\widetilde{D}$ value is considered not reliable. In this case, a fixed amount of CD $\left(D_{\text {step }}\right)$ is added to $D_{0}$ and the FDE is initialized to this new value. Then again the FDE and the B-TDE are applied (Loop 1 in the left side of the Fig. 5)

Loop 1 is iterated until $M S E<M_{1}$. As soon as this condition is fulfilled the OPM is applied and the value of $\widetilde{D}$ is compared to the threshold $\widetilde{D}_{1}$. At the same time a second check on the MSE is performed. If one of the two values is above the corresponding threshold $\left(M_{2}\right.$ and $\left.\widetilde{D}_{1}\right)$, the iterative procedure enters Loop 2 and $\widetilde{D}$ is added algebraically to the value previously set in the FDE. Loop 2 terminates only when both the conditions $\left(|\widetilde{D}|<\widetilde{D}_{1}\right.$ and MSE $\left.<M_{2}\right)$ are met and the algorithm stops. In this condition the B-TDE compensates only a minimum ISI quantity defined by the residual $D, \widetilde{D}$ the PMD and the filtering effect. Decreasing ${ }_{1}$ means that the fraction of the $D$ that is compensated by the FDE increases, but more iteration are needed.

On the other hand, if $\widetilde{D}_{1}$ is too high, the B-TDE should compensate a quantity of $C D$ greater than the value it can handle with a good accuracy. Therefore the choice should be a trade-off between accuracy of the estimation and number of iterations. The total CD compensated by the algorithm is the sum of the CD compensated by the FDE $\left(D_{M}\right)$ and the CD compensated by the B-TDE and estimated by the OPM in the last iteration $(\widetilde{D})$.

The parameters (threshold values and $D_{\text {step }}$ ) of the proposed algorithm are critical and must be properly chosen considering the characteristics of the system (bit-rate and modulation format) as we will show in the next section.

\section{EXPERIMENTAL VALIDATION}

We experimentally tested the proposed technique in a 112 Gbit/s PM-QPSK coherent test bed (Fig. 6). The output lightwave of an external cavity $\mathrm{CW}$ tunable laser $(150 \mathrm{kHz}$

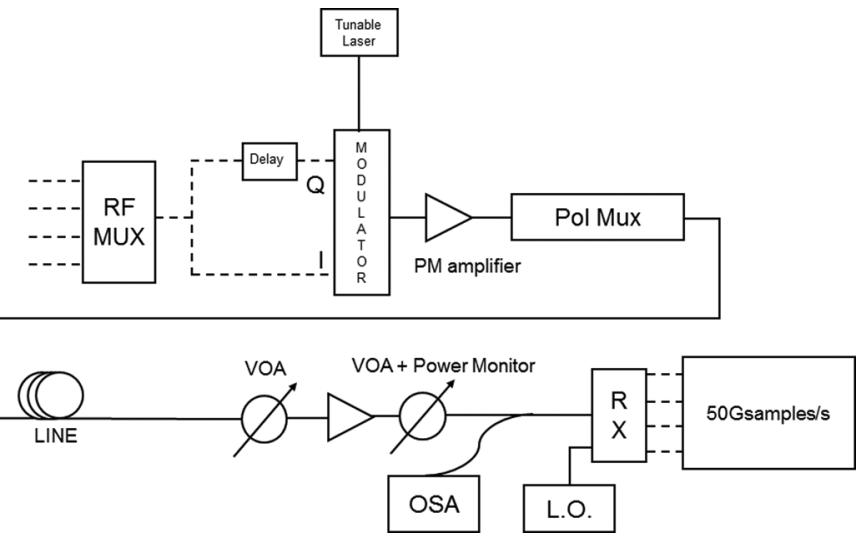

Fig. 6. Experimental set-up.

linewidth, wavelength: $\lambda=1548 \mathrm{~nm}$ ) was QPSK-modulated. Four pseudo random bit sequences (PRBS) at $7 \mathrm{Gbit} / \mathrm{s}$ were combined through a multiplexer (RF MUX). The obtained $28 \mathrm{Gbit} / \mathrm{s}$ electrical data stream was power-divided and one of the two branches was delayed by 48 symbol-times. The two RF signals were then sent to the I and Q input of the I/Q $\mathrm{LiNbO}_{3}$ modulator. The obtained $56 \mathrm{Gbit} / \mathrm{s}$ QPSK signal was then sent to a polarization multiplexer (PolMux) emulator, which split the signal into two components. One of them was delayed by 112 symbol times, rotated in polarization by $90^{\circ}$ and then coupled together to the other, thus emulating a $112 \mathrm{Gbit} / \mathrm{s}$ PM-QPSK signal. The optical signal propagated along a recirculating loop (represented by "line" in Fig. 6) including a $40 \mathrm{~km}$ Z-PLUS Fiber ${ }^{\circledR}$ span (nominal dispersion parameter equal to $20.4 \mathrm{ps} / \mathrm{nm} / \mathrm{km}$ ). The launched power was $+2 \mathrm{dBm}$. We set the loop so that the signal propagated in a range from 0 to $1000 \mathrm{~km}$ with a corresponding CD value from 0 to $20400 \mathrm{ps} / \mathrm{nm}$. No optical compensating fiber was used. At the receiver side, the signal and the local oscillator $(150 \mathrm{kHz}$ linewidth and $100 \mathrm{MHz}$ frequency offset) were sent to a polarization diversity $90^{\circ}$ hybrid coupler, and detected by four balanced receivers. The four electrical signals were sampled by a $50 \mathrm{GSample/s}$ real time oscilloscope, and then processed offline by a common PC. The receiver works using two samples-per-symbol interval. The tap coefficients of the B-TDE equalizer were used by the OPM algorithm for the CD estimation of the line.

The parameter $D_{\text {step }}$ is strictly connected to $M_{1}$ and depends on the bit-rate and the modulation format. Namely, $D_{\text {step }}$ should be chosen such that the difference between the CD compensated by the FDE and the target is less than the value manageable by the B-TDE. In order to evaluate the optimal $D_{\text {step }}$, we studied the MSE behavior at the steady-state, varying the residual uncompensated $\mathrm{CD}$, i.e., varying the dispersion compensated by the FDE $\left(D_{F D E}\right)$.

The results are reported in Fig. 7 for an OSNR of $15 \mathrm{~dB}$ and $10 \mathrm{~dB}$ and three different B-TDE number of taps $(5,11,23)$. We chose the above values of OSNR in order to study the MSE variations in a realistic condition (15 $\mathrm{dB}$ OSNR) and in a limit condition (10 dB OSNR). When the uncompensated CD is 0 , the MSE value is around $0.3 \cdot 10^{-3}$ for $15 \mathrm{~dB}$ OSNR and around 1 . $10^{-3}$ for $10 \mathrm{~dB}$ OSNR. Typically, a MSE value greater than 1.6. $10^{-3}$ indicates that the B-TDE cannot converge. As previously 


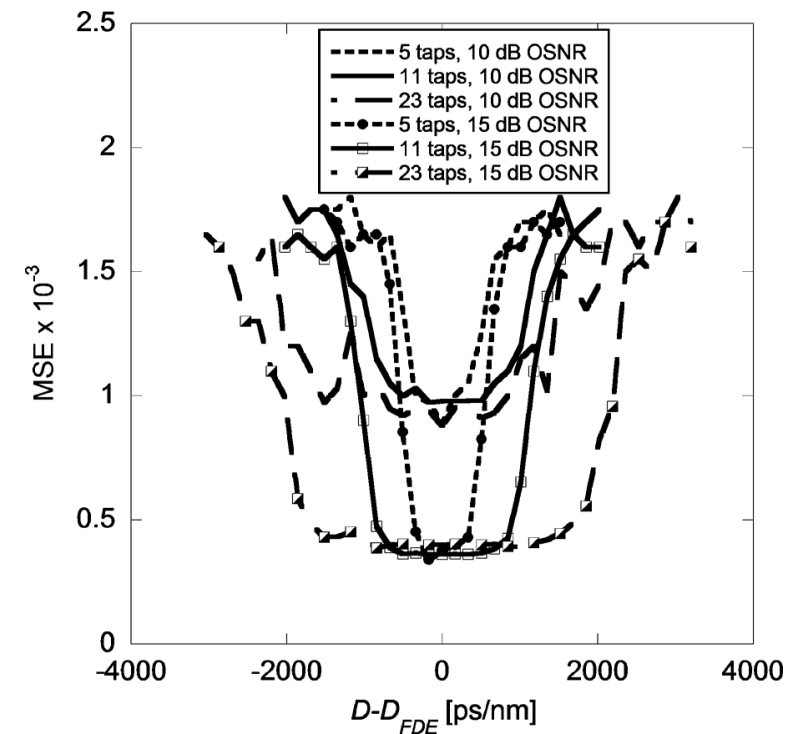

Fig. 7. MSE value vs. residual uncompensated CD using a B-TDE with 5, 11 and 23 taps for $10 \mathrm{~dB}$ OSNR.
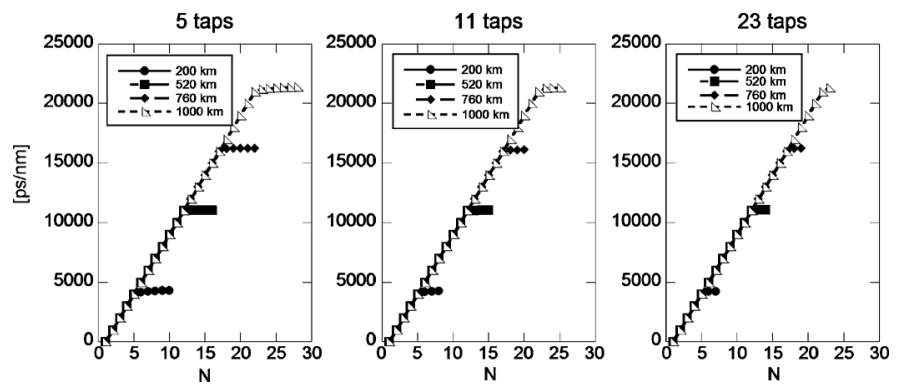

Fig. 8. $\widetilde{\boldsymbol{D}}$ vs. iterations number N.

stated, the steady-state MSE mean value is the same when the number of B-TDE taps is greater or equal the channel memory. The curves have a "bathtub" shape and their width increases with the number of taps. If the MSE comes close to $1 \cdot 10^{-3}$, the equalizer will usually converge and the OPM will give a value that, even if initially affected by a non-negligible error, will be able to make Loop 2 to converge. We chose a threshold $M_{1}=$ $1.3 \cdot 10^{-3}$ and to obtain MSE $<M_{1}$, the maximum residual uncompensated $\mathrm{CD}$ must be included between $+/-500 \mathrm{ps} / \mathrm{nm}$ for a B-TDE with only 5 taps, $+/-1000 \mathrm{ps} / \mathrm{nm}$ for a B-TDE with 11 taps and $+/-2000 \mathrm{ps} / \mathrm{nm}$ for a B-TDE with 23 taps. The difference in the MSE value between Figs. 2 and 7 is due to a different normalization. For the sake of simplicity we chose $D_{\text {step }}=1000 \mathrm{ps} / \mathrm{nm}$ in all cases, which is required for the 5 taps B-TDE. Higher value for $D_{s t e p}$ could be adopted for 11 and 23 taps; the only drawback for a small $C D_{\text {step }}$ is to increase the number of iteration in Loop 1.

The second MSE threshold $\left(M_{2}\right)$ was set to $1.1 \cdot 10^{-3}$ in order to keep a more stringent condition on the MSE but preserving good results also for the limit case of $10 \mathrm{~dB}$ OSNR. $D_{1}$ was set to $50 \mathrm{ps} / \mathrm{nm}$, in order to have a good trade-off between accuracy and number of iterations.

The dependence of the compensated and estimated $\mathrm{CD}$ value on the number of iterations, both in Loop 1 and Loop 2, is reported in Fig. 8, for 5, 11 and 23 B-TDE taps. We considered four different propagation lengths: $200 \mathrm{~km}$ (dots), $520 \mathrm{~km}$ (squares), $760 \mathrm{~km}$ (diamonds) and $1000 \mathrm{~km}$ (triangles), corresponding to 4080, 10608, 15504 and $20400 \mathrm{ps} / \mathrm{nm}$ of nominal uncompensated $D$, respectively. As described before, the iterations in Loop 1 give a fixed linear $D$ increment of $1000 \mathrm{ps} / \mathrm{nm}$ and they are represented in Fig. 8 by the points on the straight line.

The number of iterations in Loop 1 depends on $D_{0}, D_{\text {step }}$ and $M_{1}$. On the other hand the number of iterations in Loop 2 is defined by the number of B-TDE taps, by $\widetilde{D}_{1}$ and $M_{2}$. In our case the iterations in Loop 2 range between 1 (23 taps) and 7 (5 taps).

\section{Statistical ANalysis}

In the previous section the joint $\mathrm{CD}$ compensation and estimation was demonstrated on the measured data with offline processing. In order to check the statistical properties of this method we then used numerical simulations. These also allowed to assess the performance of the proposed method in presence of different optical impairments, including all-order PMD and PDL, which were not available from measured data.

We used the commercial VPI software [27] to simulate the electromagnetic field corresponding to a $112 \mathrm{Gbit} / \mathrm{s}$ PM-DQPSK NRZ transmitted sequence $\left(2^{15}-1\right.$ PRBS). This signal was then propagated on a $10 \times 100 \mathrm{~km}$ link of uncompensated G.652 fiber using the split-step Fourier method. The total accumulated dispersion was $D=16852 \mathrm{ps} / \mathrm{nm}$. The amplifier noise was added in the frequency domain; we used the model presented in [26] for PMD and a PDL element was added at each amplifier stage. The received electromagnetic field was then elaborated by means of a standard polarization-diversity balanced coherent receiver routine implemented in VPI that sampled the signal at $56 \mathrm{GHz}$ rate.

At the receiver we used an optical Gaussian band-pass filter with $40 \mathrm{GHz}$ bandwidth. Both the signal laser and the local oscillator had a linewidth of $100 \mathrm{kHz}$. There was no offset between the two laser sources and between the signal laser and the optical filter. At the receiver we used a third order Bessel filter (bandwidth of $0.7 \times$ bit rate). The OSNR was measured by the optical spectrum analyzer function of VPI. The receiver operates at an oversampling ratio equal to 2 .

We investigated eleven different conditions that are summarized as follows:

- linear propagation with OSNR $=20,15,10 \mathrm{~dB}$;

- linear propagation with OSNR $=15 \mathrm{~dB}$ and mean DGD $=10,20 \mathrm{ps}$

- linear propagation with $\mathrm{OSNR}=15 \mathrm{~dB}$ and $\mathrm{PDL}=5$, $10 \mathrm{~dB}$;

- nonlinear propagation (self-phase modulation) with $\mathrm{P}=$ - $4.4 \mathrm{dBm}(\mathrm{OSNR}=15 \mathrm{~dB})$;

- nonlinear propagation (self-phase modulation) with $\mathrm{P}=$ $10 \mathrm{dBm}($ OSNR $=28 \mathrm{~dB})$;

- nonlinear propagation with $\mathrm{P}=-4.4 \mathrm{dBm}(\mathrm{OSNR}=$ $15 \mathrm{~dB})$, mean DGD $=20 \mathrm{ps}, \mathrm{PDL}=5 \mathrm{~dB}$;

- nonlinear propagation with $\mathrm{P}=10 \mathrm{dBm}(\mathrm{OSNR}=$ $28 \mathrm{~dB}$ ), mean DGD $=20 \mathrm{ps}, \mathrm{PDL}=5 \mathrm{~dB}$.

We run 30 independent simulations for each combination of various system parameters, then we evaluated the mean value and the standard deviation of the estimation error. 

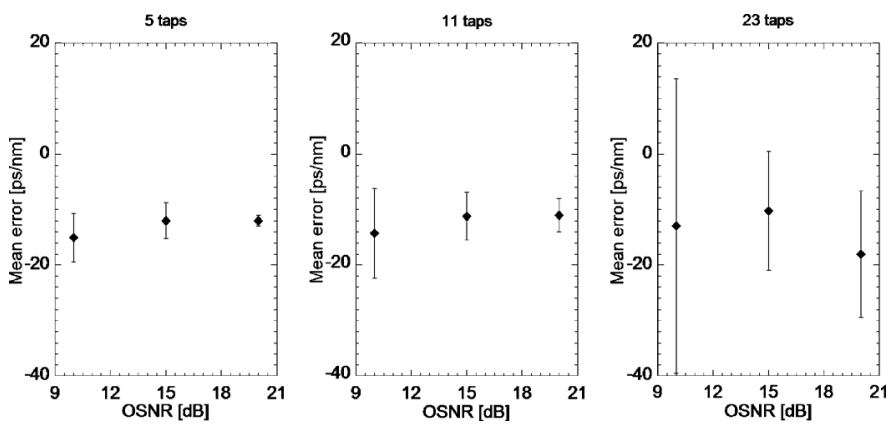

Fig. 9. CD estimation error vs. OSNR for 5 taps (left), 11 taps (center) and 23 taps (right). The error bars show $+/-$ the standard deviation.

The received samples were elaborated as described previously in Section IV. In Fig. 9 we report the CD estimation error in case of linear propagation without DGD and PMD for three different values of $\operatorname{OSNR}(10,15,20 \mathrm{~dB})$. The observed behavior was similar in all cases: the mean estimation error was less than $20 \mathrm{ps} / \mathrm{nm}$ and also the standard deviation was less than $10 \mathrm{ps} / \mathrm{nm}$ (5 and 11 taps), which are very low values in practical systems. When we used a B-TDE having 23 taps we obtained a slightly higher value of standard deviation $(27 \mathrm{ps} / \mathrm{nm})$, likely because of the more involved convergence. Due to the structure of the loop algorithm, the majority of the $\mathrm{CD}$ is compensated by the FDE and the B-TDE must compensate only the residual CD. The working condition in Fig. 9 (linear propagation without DGD and PMD and with low CD value) is such that an equalizer with a high number of taps (23 in our case) is oversized. This means that only the central taps give a significant contribution to the $\mathrm{CD}$ compensation whereas the lateral ones should be zero-valued in the ideal case. In a practical scenario, these lateral taps are not zero, but they assume small values that induce fluctuation in the estimation performed by the OPM.

We then included PMD in our simulations. In Fig. 10 we report the $\mathrm{CD}$ estimation error for a B-TDE with 11 taps in the case of linear propagation with $15 \mathrm{~dB}$ OSNR and PMD (Fig. 10(a)); 15 dB OSNR and PDL (Fig. 10(b)); nonlinear propagation (Fig. 10(c)); nonlinear propagation, DGD $=20 \mathrm{ps}$ and $\mathrm{PDL}=5 \mathrm{~dB}$ (Fig. 10(d)). The error bars represent $+/-$ the standard deviation.

In the Fig. 10(a) both the mean error and the standard deviation are very small and comparable with the data reported in Fig. 10 thus demonstrating that the PMD is not an issue.

From Fig. 10(b) it results that the PDL has a low impact on the mean value of the estimation error $(30 \mathrm{ps} / \mathrm{nm}$ for PDL $=10 \mathrm{~dB})$ but has a higher impact on the standard deviation. With PDL = $10 \mathrm{~dB}$ a standard deviation of $97 \mathrm{ps} / \mathrm{nm}$ has been obtained.

In Fig. 10(c) we report the mean error with respect to launched optical power at the beginning of each span. In these cases we activated also the non-linear part in the split step Fourier propagation software in order to include also the self-phase modulation effect. The results show that at $10 \mathrm{dBm}$ input power the mean error is higher compared with the case of $-4.4 \mathrm{dBm}$ input power but the standard deviation has a similar value in both cases.

As last test we combined together self-phase modulation, PDL and PMD (Fig. 10(d)). In particular we studied the
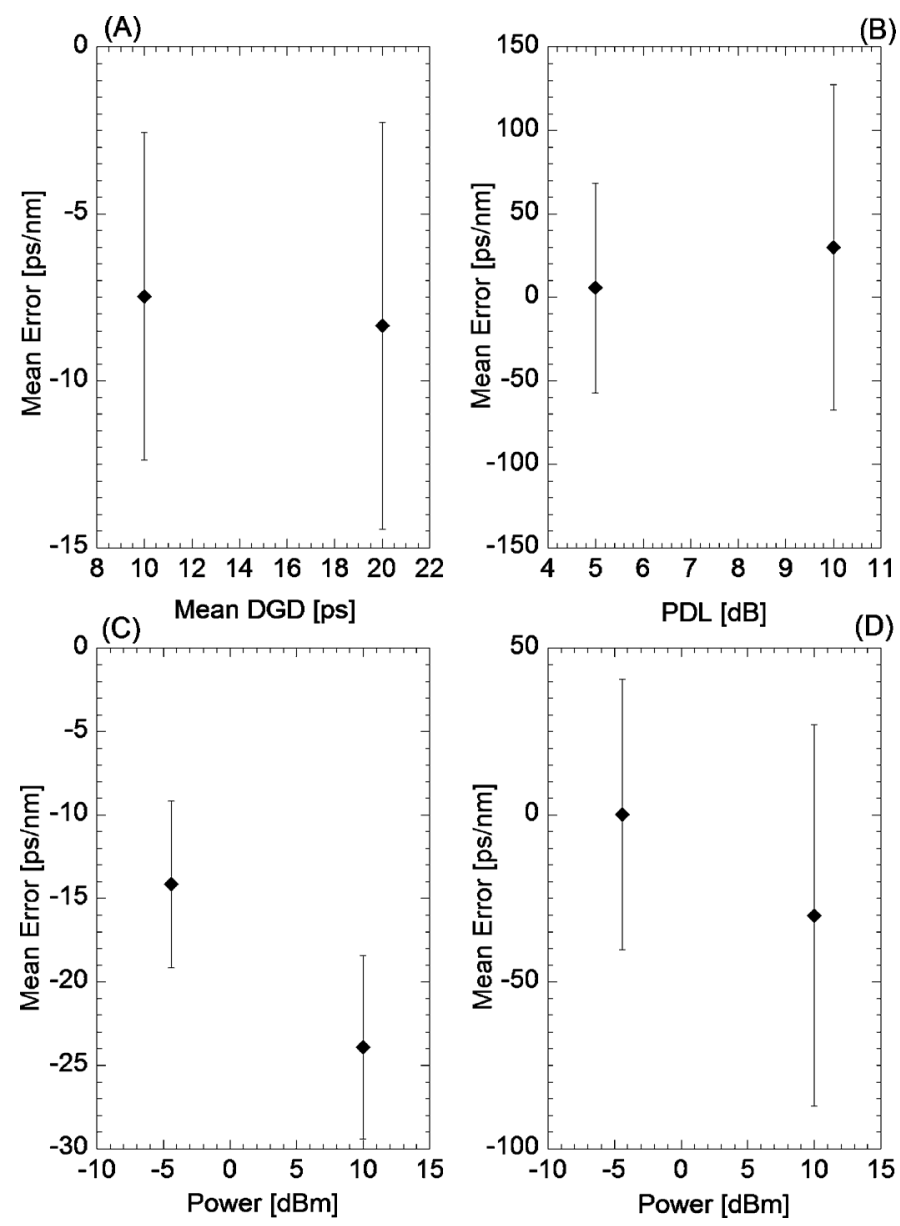

Fig. 10. CD estimation error for a 11 taps B-TDE vs. mean DGD (A); PDL (B); SPM (C); SPM, DGD and PDL (D). The error bars represent $+/-$ the standard deviation.

case with launched power $\mathrm{P}=-4.4$ and $+10 \mathrm{dBm}$, mean $\mathrm{DGD}=20 \mathrm{ps}$ and $\mathrm{PDL}=5 \mathrm{~dB}$. The maximum value for the mean error is $30 \mathrm{ps} / \mathrm{nm}$ and the maximum standard deviation is $57 \mathrm{ps} / \mathrm{nm}$ both recorded for $10 \mathrm{dBm}$ input power.

Using a B-TDE with 5 taps, the results were similar compared to the case with 11 taps. On the other hand using a B-TDE with 23 taps, the obtained results were worst compared to the case with 11 taps. The main difference was a $50 \%$ increment of the standard deviation value when the PDL was added to the system.

As can be seen, the overall performance of the proposed method is good. This is even more important since the proposed solution exploits resources already present in a system (FDE, B-TDE and the OPM) and non-dedicated to non-linear effects compensation and PDL mitigation.

Even for very high value of PDL $(10 \mathrm{~dB})$ the results are acceptable. Overall the maximum estimation error obtained in all the considered conditions was $340 \mathrm{ps} / \mathrm{nm}$. Considering that the B-TDE was not optimized for working in very stressing conditions (in particular high PDL value), the obtained values are comparable with the results recently presented [10], [28]. Furthermore the limit case with a five of taps performed very well with results comparable to the case of 11 taps and even better if compared to the case of 23 taps. This behavior can be explained considering that the majority of the $\mathrm{CD}$ is compensated by FDE 
and the B-TDE should compensate only the ISI induced by the residual uncompensated $\mathrm{CD}$, by the $\mathrm{PMD}$ and by the filtering. In case the length of the B-TDE is significantly higher compared to the channel length the convergence is more critical due to numerical fluctuations of the taps and this affects the estimation accuracy in terms of standard deviation.

\section{AlgORITHM COMPLEXITY}

We point out that any new algorithm solution, for $\mathrm{CD}$ search and compensation, brings some additional complexity, which should be evaluated. This complexity can be divided in hardware and computational. The first one is due to the use of an ASIC or a FPGA. The proposed algorithm is intended to exploit resources (FDE, B-TDE and OPM) already present in the system and thus the hardware required for $\mathrm{CD}$ search is the same used in data path. The second one can be estimated in terms of complex multiplications per iteration.

The complexity of equalizers is primarily determined by their length, which depends on the channel memory. As reported in [12] and [17], a static FDE requires about 32 complex multiplications per bit at $112 \mathrm{Gbit} / \mathrm{s}$ with $24000 \mathrm{ps} / \mathrm{nm}$ of uncompensated CD. The method presented in [16] based on adaptive FDE (filter-bank approach with time-domain cost functions) needs to iterate the algorithm for 187 times (including both first and second iteration) in order to estimate the given CD. In total 5984 complex multiplications per bit are required. In the case of the method presented in this paper, for the same amount of uncompensated CD and $D_{\text {step }}=1000 \mathrm{ps} / \mathrm{nm}$, the number of iterations in Loop 1 are 24 considering a B-TDE with 11 taps (less iterations are needed in case of an optimized $\left.D_{\text {step }}\right)$. Loop 2 must be iterated for about 10 times (we consider this number as a worst case scenario from Fig. 9) leading to a total of 34 iterations. Considering 32 and 22 complex multiplications per bit for FDE and B-TDE respectively [17], [29], a total of 1836 multiplications per transmitted bit are required for the proposed approach based on the co-operation between FDE and B-TDE. The OPM (operating only during Loop 2) can be implemented in a side-processor or micro controller in order to reduce the gate-count of the high-speed ASIC, which incorporates FDE and B-TDE. We underline that this complexity calculation deals only with the number of complex operations executed during the CD search by the FDE and B-TDE, but this is not an exhaustive evaluation of the total computational complexity.

On the other side, the pure FDE method based on a filter bank approach presented in [13] with frequency domain cost functions can be realized without the necessity of multiple FFT's. However this technique requires to filter the received signal spectrum by a range of potential CD compensation functions ( $\pm 32000 \mathrm{ps} / \mathrm{nm}$ and a step width equal to $200 \mathrm{ps} / \mathrm{nm}$ ) and consequently to compute the complex autocorrelation functions over both polarizations. Moreover the best-matching search based on clock tone information can be affected by polarization effects that are avoided using a polarization filter.

Our method combines the robust FDE-based solution with a subsequent FIR filter scan for further fine tuning. Given that the number of taps is crucial for B-TDE complexity, the proposed loop configuration permits to minimize the B-TDE taps number
(5 taps, in ideal case, and 11 taps in a real system), since it is governed only by the mean DGD, the residual CD and the filtering, regardless of the uncompensated CD of the line.

\section{CONCLUSIONS}

We proposed a solution for joint CD estimation and compensation in uncompensated links. This method does not require any prior knowledge of the link dispersion neither the use any training sequence, but it only exploits resources already present in the system (FDE, B-TDE, and OPM) and arranging them in loop configuration, with no increase in the complexity of the equalizers.

We first experimentally demonstrated the validity of the proposed solution for a maximum CD value of $20400 \mathrm{ps} / \mathrm{nm}$. We obtained very good results for a realistic case of 11 taps and for the limit cases with 23 and 5 taps. We chose 5 taps as a reference, in order to demonstrate the robustness of the loop algorithm even with a very low number of taps. In a realistic case, a higher number must be considered, in order to take into account any other kind of impairments such as low-pass filtering, frequency offset, phase noise, PMD.

In order to assess the performance of the proposed solution in terms of mean error and standard deviation, we run a set of simulations where the propagating signal was affected by different types of optical impairments (noise, PDL, PMD, selfphase modulation). The estimation results are comparable with those reported recently in the literature.

Finally, we outline that this method can be used in any DSPbased coherent system employing an FDE followed by a B-TDE aiming at inverting the linear transfer function of the channel, regardless the modulation format and the bit-rate.

\section{ACKNOWLEDGMENT}

The authors would like to thank Dr. F. Fresi for helpful discussions.

\section{REFERENCES}

[1] M. G. Taylor, "Coherent detection method using DSP for demodulation of signal and subsequent equalization of propagation impairments," IEEE Photon. Technol. Lett., vol. 16, no. 2, pp. 674-676, Feb. 2004

[2] S. J. Savory, "Digital filters for coherent optical receivers," Opt. Exp., vol. 16, no. 2, pp. 804-817, Jan. 2008.

[3] H. Sun, K.-T. Wu, and K. Roberts, "Real-time measurements of a 40 Gb/s coherent system," Opt. Exp., vol. 16, no. 2, pp. 873-879, 2008

[4] S. J. Savory, G. Gavioli, R. I. Killey, and P. Bayvel, "Digital filters for coherent optical receivers," Opt. Exp., vol. 15, no. 5, pp. 2120-2126, 2007.

[5] E. Ip and J. M. Kahn, "Digital equalization of chromatic dispersion and polarization mode dispersion," J. Lightw. Technol., vol. 25, no. 8, pp. 2033-2043, Aug. 2007.

[6] E. Ip and J. M. Kahn, "Fiber impairment compensation using coherent detection and digital signal processing," J. Lightw. Technol., vol. 28, no. 4, pp. 502-519, Feb. 2010

[7] J. Renaudier, G. Charlet, M. Salsi, O. B. Pardo, H. Mardoyan, P. Tran, and S. Bigo, "Linear fiber impairments mitigation of 40-Gbit/s polarization-multiplexed QPSK by digital processing in a coherent receiver," J. Lightw. Technol., vol. 26, no. 1, pp. 36-42, Jan. 2008.

[8] G. Colavolpe, T. Foggi, and G. Prati, "Stop-and-Go algorithm for blind equalization in QAM single-carrier coherent optical systems," IEEE Photon. Technol. Lett., vol. 22, no. 24, pp. 1838-1840, Dec. 2010.

[9] J. C. Geyer, C. R. S. Fludger, T. Duthel, C. Schulien, and B. Schmauss, "Efficient frequency domain chromatic dispersion compensation in a coherent Polmux QPSK-receiver," in Proc. OFC 2010, San Diego, CA, USA, Paper OWV5. 
[10] F. Hauske, C. Xie, Z. Zhang, C. Li, L. Li, and Q. Xiong, "Frequency domain chromatic dispersion estimation," in Proc. OFC 2010, San Diego, CA, USA, Paper JThA11.

[11] B. Spinnler, "Complexity of algorithms for digital coherent receivers," in Proc. ECOC 2009, Vienna, Austria, Paper 7.3.6.

[12] B. Spinnler, F. N. Hauske, and M. Kuschnerov, "Adaptive equalizer complexity in coherent optical receivers," in Proc. ECOC 2008, Brussels, Belgium, Paper We.2.E.4.

[13] R. A. Soriano, F. N. Hauske, N. G. Gonzalez, Z. Zhang, Y. Ye, and I. T. Monroy, "Chromatic dispersion estimation in digital coherent receivers," J. Lightw. Technol., vol. 29, no. 11, pp. 1627-1637, Jun. 2011.

[14] Q. Wang, C. Lu, A. P. T. Lau, and S. He, "Adaptive chromatic dispersion compensation for coherent communication systems using delay-tap sampling technique," IEEE Photon. Technol. Lett., vol. 23, no. 14, pp. 1016-1018, Jul. 2011.

[15] K. Ishihara, T. Kobayashi, R. Kudo, Y. Takatori, A. Sano, E. Yamada, H. Masuda, and Y. Miyamoto, "Coherent optical transmission with frequency-domain equalizers," in Proc. ECOC 2008, Brussels, Belgium, Paper We.2.E.3.

[16] M. Kuschnerov, F. N. Hauske, K. Piyawanno, B. Spinnler, A. Napoli, and B. Lankl, "Adaptive chromatic dispersion equalization for nondispersion managed coherent systems," in Proc. OFC 2009, San Diego, CA, USA, Paper OMT1.

[17] M. Kuschnerov, F. Hauske, K. Piyawanno, B. Spinnler, M. Alfiad, A. Napoli, and B. Lank1, "DSP for coherent single-carrier receivers," $J$. Lightw. Technol., vol. 27, no. 16, pp. 3614-3622, Aug. 2009.

[18] T. Xu, G. Jacobsen, S. Popov, J. Li, E. Vanin, K. Wang, A. T. Friberg, and Y. Zhang, "Chromatic dispersion compensation in coherent transmission system using digital filters," Opt. Exp., vol. 18, no. 15, pp. 16243-16257, Jul. 2010.

[19] F. N. Hauske, M. Kuschnerov, B. Spinnler, and B. Lankl, "Optical performance monitoring in digital coherent receivers," J. Lightw. Technol., vol. 27, no. 16, pp. 3623-3631, Aug. 2009.

[20] M. S. Faruk, Y. Mori, C. Zhang, K. Igarashi, and K. Kikuchi, "Multi-impairment monitoring from adaptive finite-impulse-response filters in a digital coherent receiver," Opt. Exp., vol. 18, no. 26, pp. 26929-26936, Dec. 2010.
[21] G. Colavolpe, T. Foggi, E. Forestieri, and G. Prati, "Robust multilevel coherent optical systems with linear processing at the receiver," J. Lightw. Technol., vol. 27, no. 13, pp. 2357-2369, Jul. 2009.

[22] J. G. Proakis, Digital Communications, 4th ed. New York, NY, USA: McGraw-Hill, 2001

[23] E. Forestieri and L. Vincetti, "Exact evaluation of the Jones matrix of a fiber in the presence of polarization mode dispersion of any order," J. Lightw. Technol., vol. 17, no. 12, pp. 1898-1909, Dec. 2001.

[24] J. C. Geyer, F. N. C. R. S. Hauske, Fludger, T. Duthel, C. Schulien, M. Kuschnerov, K. Piyawanno, D. van den Borne, E.-D. Schmidt, B. Spinnler, H. de Waardt, B. Lankl, and B. Schmauss, "Channel parameter estimation for polarization diverse coherent receivers," IEEE Photon. Technol. Lett., vol. 20, no. 10, pp. 776-778, May 2008.

[25] D. Van den Borne, T. Duthel, C. Fludger, E.-D. Schmidt, T. Wuth, C. Schulien, E. Gottwald, G. Khoe, and H. de Waardt, "Coherent equalization versus direct detection for $111-\mathrm{Gb} / \mathrm{s}$ ethernet transport," in Proc. Dig. LEOS Summer Top. Meetings, 2007, pp. 11-12, Paper MA2.4.

[26] D. Marcuse, C. R. Menyuk, and P. K. A. Wai, "Application of the Manakov-PMD equation to studies of signal propagation in optical fibers with randomly varying birefringence," J. Lightw. Technol., vol. 15, no. 9, pp. 1735-1746, 1997.

[27] [Online]. Available: http://www.vpiphotonics.com/

[28] Q. Sui, A. P. T. Lau, and C. Lu, "Fast and robust chromatic dispersion estimation using auto-correlation of signal power waveform for DSP based-coherent systems," in Proc. OFC/NFOEC 2012, Los Angeles, CA, USA, Paper OW4G.3.

[29] B. Spinnler, "Equalizer design and complexity for digital coherent receivers," IEEE J. Sel. Topics Quantum Electron., vol. 16, no. 5, pp. 1180-1192, Sep.-Oct. 2010.

Author biographies not included by author request due to space constraints. 